\title{
Correction to: System Identification and Control Design of a Wireless Charging Transfer System with Double-Sided LCC Converter
}

\author{
Ahmad Siroos $^{1} \cdot$ Mostafa Sedighizadeh $^{1}$ (1) $\cdot$ Ebrahim $^{\text {Afjei }}{ }^{1} \cdot$ Alireza Sheikhi Fini $^{2} \cdot$ Somayeh Yarkarami $^{3}$
}

Published online: 2 June 2021

(c) King Fahd University of Petroleum \& Minerals 2021

Correction to: Arabian Journal for Science and Engineering
https://doi.org/10.1007/s13369-021-05548-0

In this erratum, we correct a mistake about Fig. 10 that appears in [1]. Figure 10 appeared in [1] is not our experimental setup and we had not received permission for using it. The following figure shows the corrected form of Fig. 10. The authors would like to apologize for any inconvenience caused.

\section{Reference}

Siroos, A., Sedighizadeh, M., Afjei, E., Fini, A.S., Yarkarami, S.: System identification and control design of a wireless charging transfer system with double-sided LCC converter. Arab. J. Sci. Eng. (2021).

The original article can be found online at https://doi.org/10.1007/ s13369-021-05548-0.

Mostafa Sedighizadeh

m_sedighi@sbu.ac.ir

1 Faculty of Electrical Engineering, Shahid Beheshti University, Evin, Tehran, Iran

2 Power System Operation and Planning Research Department, Niroo Research Institute, Tehran, Iran

3 Department of Electrical Engineering, West Tehran Branch, Islamic Azad University, Tehran, Iran 

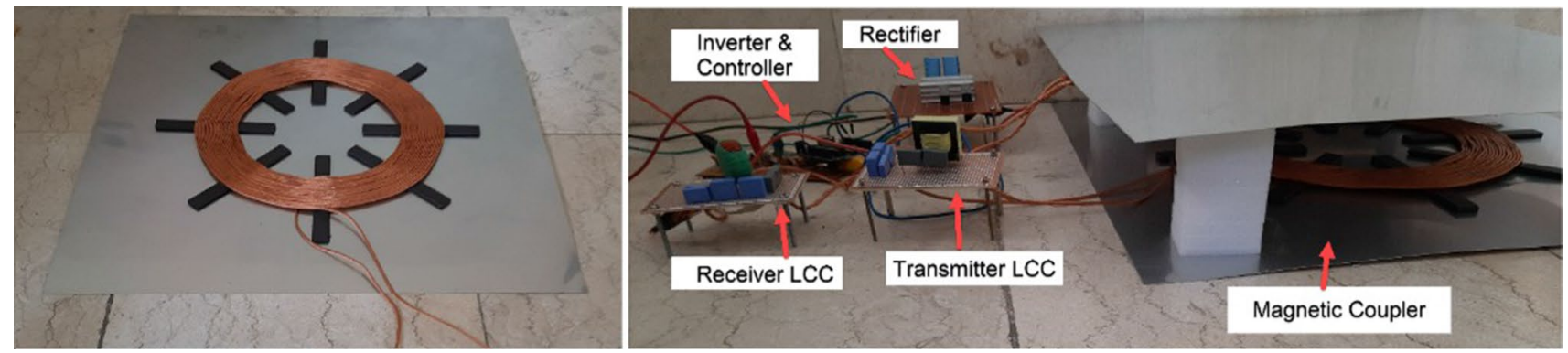

Fig. 10 Experimental setup of the WPT system 